\title{
Comparisons of Predictions from Exact Amplitude-Based Resummation Methods with LHC and Cosmological Data
}

\author{
B.F.L. Ward ${ }^{* \dagger}$ \\ Department of Physics, Baylor University \\ E-mail:bfl_ward@baylor.edu
}

\section{S.K. Majhi}

Department of Theoretical Physics, Indian Association for the Cultivation of Science

E-mail: tpskm@iacs.res.in

\section{A. Mukhopadhyay}

Department of Physics, Baylor University

E-mail: Aditi_Mukhopadhyay@baylor.edu

\section{S.A. Yost}

Department of Physics, The Citadel

E-mail: scott.yostecitadel.edu

\begin{abstract}
We present the current status of the comparisons with the respective data of the predictions of our approach of exact amplitude-based resummation in quantum field theory in two areas of investigation: precision QCD calculations of all four of us as needed for LHC physics and the resummed quantum gravity realization by one of us (B.F.L.W.) of Einstein's theory of general relativity as formulated by Feynman. The agreement between the theoretical predictions and the data exhibited continues to be encouraging.
\end{abstract}

11th International Symposium on Radiative Corrections (Applications of Quantum Field Theory to Phenomenology)

22-27 September 2013

Lumley Castle Hotel, Durham, UK

\footnotetext{
* Speaker.

${ }^{\dagger}$ Work supported in part by D.o.E. grant DE-FG02-09ER41600.

†Work supported in part by D.o.E. grant DE-PS02-09ER09-01 and grants from The Citadel Foundation.
} 


\section{Introduction}

Successful running of the LHC during 2010-2012 has allowed the accumulation of large samples of data on SM standard candle processes such as heavy gauge boson production and decay to lepton pairs: for example, samples exceeding $10^{7}$ events for $Z / \gamma^{*}$ production and decay to $\ell \bar{\ell}, \ell=e, \mu$, now exist for ATLAS and CMS. Such data emphasize that the era of precision QCD, wherein one needs predictions for QCD processes at the total precision tag of $1 \%$ or better, has arrived. Its arrival makes more manifest the need for exact, amplitude-based resummation of large higher order effects; for, with such resummation one may have better than $1 \%$ precision as a realistic goal as we shall show in what follows. Such precision allows one to distinguish new physics(NP) from higher order SM processes and to distinguish different models of new physics from one another as well. In an analogous development, one of us(B.F.L.W.) has shown that the extension of the exact amplitude-based resummation approach to Einstein's theory of general relativity allows one to make contact with UV sensitive cosmological data using ordinary quantum field theoretic methods. Here, we present the status of these two applications of exact amplitude-based resummation theory in quantum field theory in relation to recent available data from the LHC and from cosmological observations.

Our discussion proceeds as follows. First, we review the elements our approach to precision LHC physics, an amplitude-based $\mathrm{QED} \otimes \mathrm{QCD}(\equiv \mathrm{QCD} \otimes \mathrm{QED})$ exact resummation theory [1] realized by MC methods. We start from the well-known fully differential representation

$$
d \sigma=\sum_{i, j} \int d x_{1} d x_{2} F_{i}\left(x_{1}\right) F_{j}\left(x_{2}\right) d \hat{\sigma}_{\mathrm{res}}\left(x_{1} x_{2} s\right)
$$

of a hard LHC scattering process, where $\left\{F_{j}\right\}$ and $d \hat{\sigma}_{\text {res }}$ are the respective parton densities and reduced hard differential cross section and we indicate that the latter has been resummed for all large EW and QCD higher order corrections in a manner consistent with achieving a total precision tag of $1 \%$ or better for the total theoretical precision of (1.1). The determination of the total theoretical precision $\Delta \sigma_{\text {th }}$ of (1.1) is central to precision QCD theory. It can be decomposed into its physical and technical components as defined in Refs. [2,3]. Knowledge of $\Delta \sigma_{\text {th }}$ is essential to the faithful application of any theoretical prediction to precision experimental data for new physics signals, SM backgrounds, and over-all normalization considerations. In general, if $\Delta \sigma_{\text {th }} \leq f \Delta \sigma_{\text {expt }}$, where $\Delta \sigma_{\text {expt }}$ is the respective experimental error and $f \lesssim \frac{1}{2}$, the theoretical uncertainty will not significantly affect the analysis of the data for physics studies in an adverse way. It was with the goal of achieving such a provable theoretical precision tag that we have developed the QCD $\otimes$ QED resummation theory in Refs. [1] for all components of (1.1). The master formula for the starting point in all cases is

$$
\begin{gathered}
d \bar{\sigma}_{\mathrm{res}}=e^{\mathrm{SUM}_{\mathrm{IR}}(\mathrm{QCED})} \sum_{n, m=0}^{\infty} \frac{1}{n ! m !} \int \prod_{j_{1}=1}^{n} \frac{d^{3} k_{j_{1}}}{k_{j_{1}}} \\
\prod_{j_{2}=1}^{m} \frac{d^{3} k_{j_{2}}^{\prime}}{k_{j_{2}}^{\prime}} \int \frac{d^{4} y}{(2 \pi)^{4}} e^{i y \cdot\left(p_{1}+q_{1}-p_{2}-q_{2}-\sum k_{j_{1}}-\sum k_{j_{2}}^{\prime}\right)+D_{\mathrm{QCED}}} \\
\tilde{\bar{\beta}}_{n, m}\left(k_{1}, \ldots, k_{n} ; k_{1}^{\prime}, \ldots, k_{m}^{\prime}\right) \frac{d^{3} p_{2}}{p_{2}^{0}} \frac{d^{3} q_{2}}{q_{2}^{0}}
\end{gathered}
$$

where $d \bar{\sigma}_{\text {res }}$ is either the reduced cross section $d \hat{\sigma}_{\text {res }}$ or the differential rate associated to a DGLAPCS $[4,5]$ kernel involved in the evolution of the $\left\{F_{j}\right\}$ and where the new (YFS-style [6,7]) nonAbelian residuals $\tilde{\bar{\beta}}_{n, m}\left(k_{1}, \ldots, k_{n} ; k_{1}^{\prime}, \ldots, k_{m}^{\prime}\right)$ have $n$ hard gluons and $m$ hard photons and we show 
the generic $2 f$ final state with momenta $p_{2}, q_{2}$ for definiteness. The infrared functions $\operatorname{SUM}_{\mathrm{IR}}(\mathrm{QCED})$, $D_{\mathrm{QCED}}$ are given in Refs. $[1,8,9]$. The result (1.2) is exact and its residuals $\tilde{\bar{\beta}}_{n, m}$ allow a rigorous parton shower/ME matching via their shower-subtracted counterparts $\hat{\overline{\tilde{\beta}}}_{n, m}[1]$.

Using the result (1.2), one of us(B.F.L.W.) has shown in Ref. [10] that an exact, amplitudebased resummation approach to Feynman's formulation of Einstein's theory is possible via the following representation of the Feynman propagators in that theory:

$$
\begin{aligned}
i \Delta_{F}^{\prime}(k) & =\frac{i}{\left(k^{2}-m^{2}-\Sigma_{s}+i \varepsilon\right)} \\
& =\frac{i e^{B_{g}^{\prime \prime}(k)}}{\left(k^{2}-m^{2}-\Sigma_{s}^{\prime}+i \varepsilon\right)} \\
& \left.\equiv i \Delta_{F}^{\prime}(k)\right|_{\text {resummed }} .
\end{aligned}
$$

for scalar fields with an attendant generalization for spinning fields [10]. We stress that (1.3) is exact. $B_{g}^{\prime \prime}(k)$ is given in Refs. [10] and is presented below.

We now discuss in turn the two paradigms opened by (1.2) for precision QCD for the LHC and for exact resummation of Einstein's theory in the context of comparisons with recent data.

\section{Precision QCD for the $\mathrm{LHC}$ in Comparison to Data}

We first recall that, as we have shown in Refs. [11], the methods we employ for resummation of the QCD theory are fully consistent with the methods in Refs. [12,13]. A key difference between our approach and the two in Refs. $[12,13]$ is that our approach is exact whereas the latter approaches are approximate: in Refs. [12], the observation that, for any integrable function $f(z)$,

$$
\left|\int_{0}^{1} d z z^{n-1} f(z)\right| \leq\left(\frac{1}{n}\right) \max _{z \in[0,1]}\{|f(z)|\}
$$

is used to drop non-singular terms in the cross section at $z \rightarrow 1$, the respective threshold point, in going to n-Mellin space to resum the respective large threshold effects; in Refs. [13], terms of $\mathscr{O}(\lambda)$ for $\lambda=\sqrt{\Lambda / Q}$ are dropped, where $\lambda \sim 0.3 \mathrm{GeV}$ and $Q \sim 100 \mathrm{GeV}$, so that $\lambda \simeq 5.5 \%$. The known equivalence of the two approaches in Refs. [12,13] implies that a similar error holds for the approach in Refs. [12]. These two approaches may be used to construct approximations to our residuals $\tilde{\bar{\beta}}_{n, 0}$. We will pursue such approximations elsewhere [14].

Similarly, we show in the fourth paper in Refs. [11] that the approach to resummation in Refs. [15], which is realized in the MC integration program ResBos [16] and which, for the case of heavy gauge boson production in hadron colliders is presented from the fourth paper in Refs. [15] as

$$
\begin{gathered}
\frac{d \sigma}{d Q^{2} d y d Q_{T}^{2}} \sim \frac{4 \pi^{2} \alpha^{2}}{9 Q^{2} s}\left\{\int \frac{d^{2} b}{(2 \pi)^{2}} e^{i \vec{Q}_{T} \cdot \vec{b}} \sum_{j} e_{j}^{2} \widetilde{W}_{j}\left(b^{*} ; Q, x_{A}, x_{B}\right) e^{\left\{-\ln \left(Q^{2} / Q_{0}^{2}\right) g_{1}(b)-g_{j / A}\left(x_{A}, b\right)-g_{j / B}\left(x_{B}, b\right)\right\}}\right. \\
\left.+Y\left(Q_{T} ; Q, x_{A}, x_{B}\right)\right\}
\end{gathered}
$$

is also approximate at the several \% level, where we have the usual kinematics so that $\vec{Q}_{T}=\vec{p}_{T}$ is the $\gamma *$ transverse momentum, A,B are protons at the LHC, $s$ is the cms squared energy of the 
protons, $Q^{\mu}$ is the $\gamma * 4$-momentum so that $Q^{2}$ is the $\gamma *$ mass squared, and $y=\frac{1}{2} \ln \left(Q^{+} / Q^{-}\right)$is the $\gamma *$ rapidity so that $x_{A}=e^{y} Q / \sqrt{s}$ and $x_{B}=e^{-y} Q / \sqrt{s}$. We have in mind that $Q$ is near $M_{Z}$ here. In (2.1), the term involving the $\widetilde{W}_{j}$ carries the effects from QCD resummation as developed in Refs. [15] and the $Y$ term includes those contributions which are 'regular' at $Q_{T}=p_{T} \rightarrow 0$ in the sense of Refs. [15], i.e., order by order in perturbation theory they are derived from the parts of the attendant hard scattering coefficients that are less singular than $Q_{T}^{-2} \times(\operatorname{logs}$ or 1$)$ or $\delta\left(\vec{Q}_{T}\right)$ as $Q_{T}=p_{T} \rightarrow 0$. We refer the reader to Refs. [15] for the remaining notations in (2.1). Our question concerns the physical precision of the term involving the $\widetilde{W}_{j}$; for, the $Y$ term is perturbative and can be computed in principle to the required accuracy by the standard methods. What we note in the fourth paper in Ref. [11] is that the resummed term drops terms $\mathscr{O}\left(Q_{T} / Q\right)$ in all orders of $\alpha_{s}$. For example, at $Q_{T}=5 \mathrm{GeV}$ and $Q=M_{Z}$ this gives a $5.5 \%$ physical precision error(PPE). We also note [11] that the errors on the non-perturbative functions $g_{\ell}$ yield a $\sim 1.5 \%$ PPE. Evidently, this approach to resummation is not precise enough for the $1 \%$ precision tag that we seek with our approach in (1.2); it may be used to give approximations to our new residuals $\tilde{\bar{\beta}}_{m, n}$ for qualitative studies of consistency, for example. We will address such matters elsewhere [14].

Focussing on the DGLAP-CS theory itself and applying the formula in (1.2) to the calculation of the kernels, $P_{A B}$, we arrive at an improved IR limit of these kernels. In this IR-improved DGLAPCS theory $[8,9]$ large IR effects are resummed for the kernels themselves. The resulting new resummed kernels, $P_{A B}^{e x p}[8,9]$, yield a new resummed scheme for the PDF's and the reduced cross section:

$$
\begin{aligned}
F_{j}, \hat{\sigma} \rightarrow F_{j}^{\prime}, \hat{\sigma}^{\prime} \text { for } \\
P_{g q}(z) \rightarrow P_{g q}^{\exp }(z)=C_{F} F_{Y F S}\left(\gamma_{q}\right) e^{\frac{1}{2}} \delta_{q} \frac{1+(1-z)^{2}}{z} z^{\gamma_{q}}, \text { etc. }
\end{aligned}
$$

This new scheme gives the same value for $\sigma$ in (1.1) with improved MC stability as discussed in Ref. [11]. Here, the YFS [6] infrared factor is given by $F_{Y F S}(a)=e^{-C_{E} a} / \Gamma(1+a)$ where $C_{E}$ is Euler's constant and we refer the reader to Ref. [8,9] for the definition of the infrared exponents $\gamma_{q}, \delta_{q}$ as well as for the complete set of equations for the new $P_{A B}^{e x p} . C_{F}$ is the quadratic Casimir invariant for the quark color representation.

The basic physical idea underlying the new kernels, which was already shown by Bloch and Nordsieck [17], is illustrated in Fig. 1: the coherent state of very soft massless quanta of the

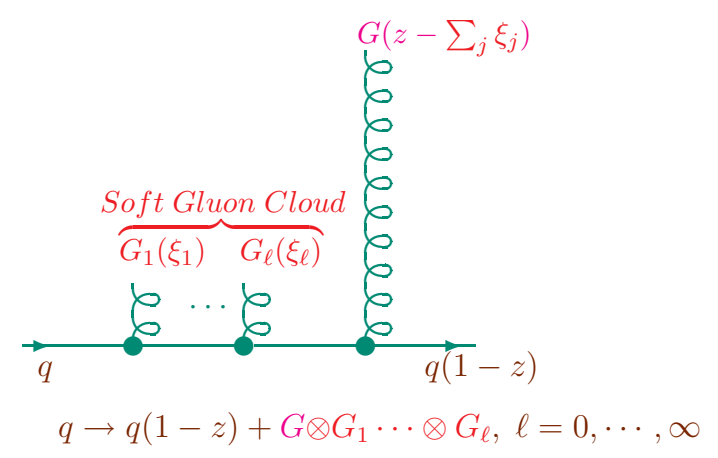

Figure 1: Bloch-Nordsieck soft quanta for an accelerated charge. 
respective gauge field generated by an accelerated charge makes it impossible to know which of the infinity of possible states one has made in the splitting process $q(1) \rightarrow q(1-z)+G \otimes G_{1} \cdots \otimes$ $G_{\ell}, \ell=0, \cdots, \infty$ illustrated in Fig. 1. The new kernels take this effect into account: they resum terms $\mathscr{O}\left(\left(\alpha_{s} \ln \left(q^{2} / \Lambda^{2}\right) \ln (1-z)\right)^{n}\right)$ for the IR limit $z \rightarrow 1$ to generate the Gribov-Lipatov exponents $\gamma_{A}$ which therefore start in $\mathscr{O}(\hbar)$ in the loop expansion. See Refs. [11] for a calculation of the $\gamma_{A}$.

The new MC Herwiri1.031 [11] gives the first realization of the new IR-improved kernels in the Herwig6.5 [18] environment. We are in the process of realizing the new kernels in the Herwig++ [19], Pythia8 [20], Sherpa [21] and Powheg [22] environments as well. Here, we illustrate in Fig. 2 some of the recent comparisons we have made between Herwiri1.031 and Herwig6.510, both with and without the MC@NLO [23] exact $\mathscr{O}\left(\alpha_{s}\right)$ correction, in relation to the LHC data [24,25] on $Z / \gamma *$ production with decay to lepton pairs ${ }^{1}$. Just as we found in Refs. [11]

(a)

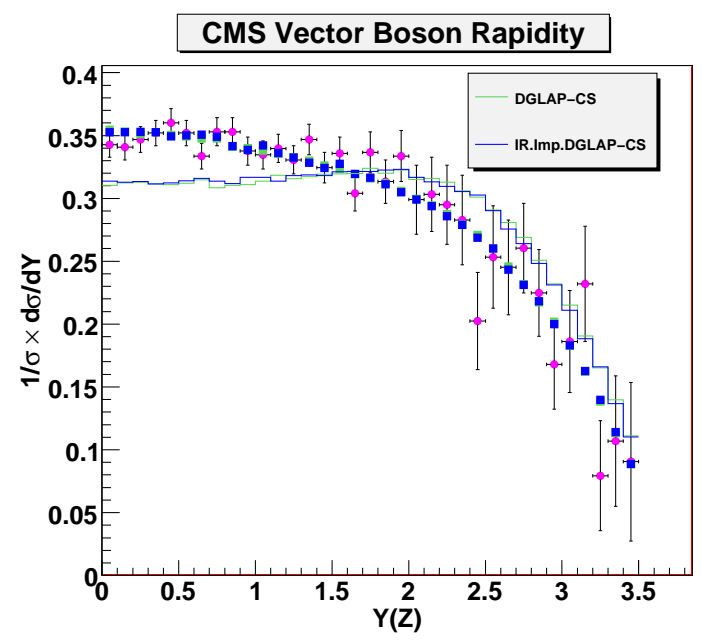

(b)

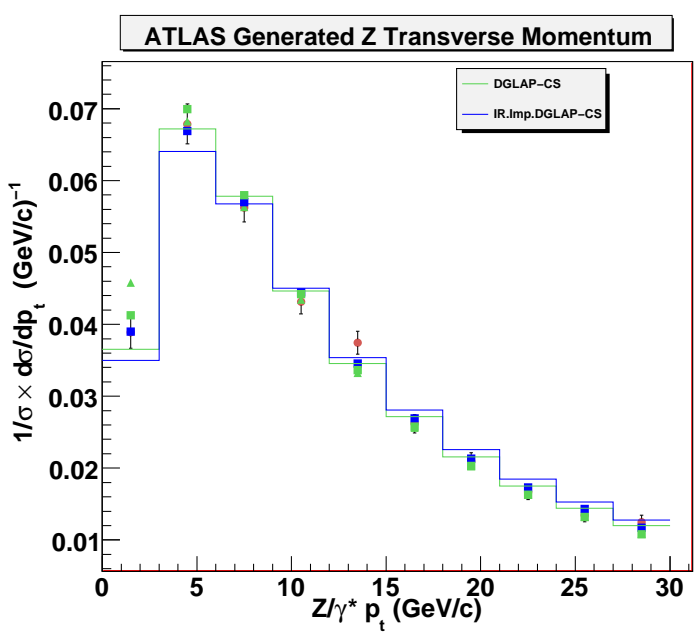

Figure 2: Comparison with LHC data: (a), CMS rapidity data on $\left(Z / \gamma^{*}\right)$ production to $e^{+} e^{-}, \mu^{+} \mu^{-}$ pairs, the circular dots are the data, the green(blue) lines are HERWIG6.510(HERWIRI1.031); (b), ATLAS $p_{T}$ spectrum data on $\left(Z / \gamma^{*}\right)$ production to (bare) $e^{+} e^{-}$pairs, the circular dots are the data, the blue(green) lines are HERWIRI1.031(HERWIG6.510). In both (a) and (b) the blue(green) squares are MC@NLO/HERWIRI1.031(HERWIG6.510(PTRMS $=2.2 \mathrm{GeV})$ ). In $(\mathrm{b})$, the green triangles are MC@NLO/HERWIG6.510(PTRMS =0). These are otherwise untuned theoretical results.

for the FNAL data on single $Z / \gamma^{*}$ production, the unimproved MC requires the very hard value of PTRMS $\cong 2.2 \mathrm{GeV}$ to give a good fit to the $p_{T}$ spectra as well as the rapidity spectra whereas the IR-improved calculation gives very good fits to both of the spectra without the need of such a hard value of PTRMS, the rms value for an intrinsic Gaussian $p_{T}$ distribution, for the proton wave function: the $\chi^{2} /$ d.o.f are respectively $(0.72,0.72),(1.37,0.70),(2.23,0.70)$ for the $p_{T}$ and rapid-

\footnotetext{
${ }^{1}$ Similar comparisons were made in relation to such data $[26,27]$ from FNAL in Refs. [11] and we comment presently on the connection between the two sets of comparisons.
} 
ity data for the MC@NLO/HERWIRI1.031, MC@NLO/HERWIG6.510(PTRMS = 2.2GeV) and MC@NLO/HERWIG6.510(PTRMS =0) results. Such a hard intrinsic value of PTRMS contradicts the results in Refs. [28, 29], as we discuss in Refs. [11]. To illustrate the size of the exact $\mathscr{O}\left(\alpha_{s}\right)$ correction, we also show the results for both Herwig6.510(green line) and Herwiri1.031(blue line) without it in the plots in Fig. 2. As expected, the exact $\mathscr{O}\left(\alpha_{s}\right)$ correction is important for both the $p_{T}$ spectra and the rapidity spectra. The suggested accuracy at the $10 \%$ level shows the need for the NNLO extension of MC@NLO, in view of our goals for this process. We also note that, with the $1 \%$ precision goal, one also needs per mille level control of the EW corrections. This issue is addressed in the new version of the $\mathscr{K} \mathscr{K}$ MC [30], version 4.22, which now allows for incoming quark antiquark beams - see Ref. [30] for further discussion of the relevant effects in relation to other approaches [31].

As we show in Refs. [11], one may use the new precision data at ATLAS and CMS, where one has now more than $10^{7} Z / \gamma *$ decays to lepton pairs per experiment, to distinguish between the fundamental description in Herwiri1.031 and the ad hocly hard intrinsic $p_{T}$ in Herwig6.5 by comparing the data to the predictions of the detailed line shape and of the more finely binned $p_{T}$ spectra - see Figs. 3 and 4 in the last two papers in Refs. $[11]^{2}$. We await the releases of the new precision data accordingly.

\section{Resummed Quantum Gravity: Comparison with Data}

One of us(B.F.L.W.), using his application of exact amplitude-based resummation theory to Feynman's formulation of Einstein's theory, as described in Refs. [10], has arrived in Ref. [33] at a first principles prediction of the cosmological constant that is close to the observed value [34,35], $\rho_{\Lambda} \cong\left(2.368 \times 10^{-3} \mathrm{eV}(1 \pm 0.023)\right)^{4}$. We now recapitulate this promising result and some of the cross checks that it has passed.

Using the deep UV result

$$
B_{g}^{\prime \prime}(k)=\frac{\kappa^{2}\left|k^{2}\right|}{8 \pi^{2}} \ln \left(\frac{m^{2}}{m^{2}+\left|k^{2}\right|}\right),
$$

it is shown in Ref. [33] that the UV limit of Newton's constant, $G_{N}(k)$, is given by

$$
g_{*}=\lim _{k^{2} \rightarrow \infty} k^{2} G_{N}\left(k^{2}\right)=\frac{360 \pi}{c_{2, e f f}} \cong 0.0442
$$

where $[10,33] c_{2, e f f} \cong 2.56 \times 10^{4}$ for the known world. The same formula (3.1) allows one to show [33] that the contribution of a scalar field to $\Lambda$ is

$$
\Lambda_{s}=-8 \pi G_{N} \frac{\int d^{4} k}{2(2 \pi)^{4}} \frac{\left(2 k_{0}^{2}\right) e^{-\lambda_{c}\left(k^{2} /\left(2 m^{2}\right)\right) \ln \left(k^{2} / m^{2}+1\right)}}{k^{2}+m^{2}} \cong-8 \pi G_{N}\left[\frac{1}{G_{N}^{2} 64 \rho^{2}}\right]
$$

where $\rho=\ln \frac{2}{\lambda_{c}}$ for $\lambda_{c}=\frac{2 m^{2}}{\pi M_{P l}^{2}}$ and we have used the calculus of Refs. [10,33]. We note that standard methods [33] then allow one to show that a Dirac fermion contributes -4 times $\Lambda_{s}$ to $\Lambda$, so that the

\footnotetext{
${ }^{2}$ We note that already in Refs. [32] the discriminating power of $p_{T}$ spectra in single $Z / \gamma^{*}$ production at the LHC among theoretical predictions is manifest - see the last paper in Refs. [11] for more discussion on this point.
} 
deep UV limit of $\Lambda$ becomes

$$
\begin{aligned}
& \Lambda(k) \underset{k^{2} \rightarrow \infty}{\longrightarrow} k^{2} \lambda_{*}, \\
& \lambda_{*}=-\frac{c_{2, e f f}}{2880} \sum_{j}(-1)^{F_{j}} n_{j} / \rho_{j}^{2} \cong 0.0817
\end{aligned}
$$

where $F_{j}$ is the fermion number of $j$ and $\rho_{j}=\rho\left(\lambda_{c}\left(m_{j}\right)\right)$. Our results for $\left(g_{*}, \lambda_{*}\right)$ agree qualitatively with those in Refs. [36,37]. Indeed, as we show in Ref. [33], there is no disagreement in principle between our gauge invariant, cut-off independent results and the gauge dependent, cut-off dependent results in Refs. [36,37].

\subsection{A Resummed Quantum Gravity Estimate of $\Lambda$}

Toward obtaining an estimate the value of $\Lambda$ today, we make use of the normal-ordered form of Einstein's equation,

$$
: G_{\mu v}:+\Lambda: g_{\mu v}:=-8 \pi G_{N}: T_{\mu v}:
$$

From the coherent state representation of the thermal density matrix one then arrives at the Einstein equation in the form of thermally averaged quantities with $\Lambda$ given by our result above in lowest order. Using the result from Refs. [37] that the transition time between the Planck regime and the classical Friedmann-Robertson-Walker(FRW) regime is $t_{t r} \sim 25 t_{P l}$, we introduce

$$
\rho_{\Lambda}\left(t_{t r}\right) \equiv \frac{\Lambda\left(t_{t r}\right)}{8 \pi G_{N}\left(t_{t r}\right)}=\frac{-M_{P l}^{4}\left(k_{t r}\right)}{64} \sum_{j} \frac{(-1)^{F} n_{j}}{\rho_{j}^{2}}
$$

and follow the arguments in Refs. [38] ( $t_{e q}$ is the time of matter-radiation equality) to get

$$
\begin{aligned}
& \rho_{\Lambda}\left(t_{0}\right) \cong \frac{-M_{P l}^{4}\left(1+c_{2, e f f} k_{t r}^{2} /\left(360 \pi M_{P l}^{2}\right)\right)^{2}}{64} \sum_{j} \frac{(-1)^{F} n_{j}}{\rho_{j}^{2}} \\
& \quad \times\left[\frac{t_{t r}^{2}}{t_{e q}^{2}} \times\left(\frac{t_{e q}^{2 / 3}}{t_{0}^{2 / 3}}\right)^{3}\right] \\
& \cong \frac{-M_{P l}^{2}(1.0362)^{2}\left(-9.197 \times 10^{-3}\right)}{64} \frac{(25)^{2}}{t_{0}^{2}} \\
& \cong\left(2.400 \times 10^{-3} \mathrm{eV}\right)^{4} .
\end{aligned}
$$

where we take the age of the universe to be $t_{0} \cong 13.7 \times 10^{9}$ yrs. In (3.7), the first factor in the square bracket comes from the period from $t_{t r}$ to $t_{e q}$ (radiation dominated) and the second factor comes from the period from $t_{e q}$ to $t_{0}$ (matter dominated) ${ }^{3}$. This estimate should be compared with the experimental result $\left.[34,35]^{4} \rho_{\Lambda}\left(t_{0}\right)\right|_{\text {expt }} \cong\left(2.368 \times 10^{-3} \mathrm{eV}(1 \pm 0.023)\right)^{4}$.

In Ref. [33], it is shown that the result in (3.7) is robust to the corrections associated with the EW, QCD chiral and GUT suymmetry breaking scales, as these are suppressed by a factor $\sim \mu_{\text {Breaking }}{ }^{4} /\left(.01 M_{P l}^{4}\right)$ if the respective breaking scale is $\mu_{\text {Breaking. }}$. It is also shown in Ref. [33] that continuity of the Hubble parameter across the boundary from the Planck regime to the FRW regime

\footnotetext{
${ }^{3}$ The operator field method forces the vacuum energies to follow the same scaling as the non-vacuum excitations.

${ }^{4}$ See also Ref. [39] for an analysis that suggests a value for $\rho_{\Lambda}\left(t_{0}\right)$ that is qualitatively similar to this result.
} 
in the model of Ref. [37] requires a gauge transformation, which, if taken as a dilatation, shows that the result in (3.7) leads to the value $\Omega_{\Lambda}\left(t_{\mathrm{BBN}}\right) \cong 1.31 \times 10^{-3}$, so that it does not significantly affect Big Bang Nucleosynthesis(BBN) [40], where we use standard notation. The presence of possible higher degrees of freedom such as those in susy GUT models [41] is discussed in Ref. [33] with the conclusion that such models are consistent with (3.7) only if they are modified with new degrees of freedom at scales much higher than the EW scale - see Ref. [33] for the detailed discussion. Finally, we note [33], concerning the issue of the covariant conservation of matter in the current universe, that only when $\dot{\Lambda}+8 \pi \dot{G}_{N}=0$ holds is this guaranteed and that violations of such conservation are allowed as long as they are small, as discussed in Refs. [42].

In closing, two of us (B.F.L.W., S.A.Y.) thank Prof. Ignatios Antoniadis for the support and kind hospitality of the CERN TH Unit while part of this work was completed.

\section{References}

[1] C. Glosser, S. Jadach, B.F.L. Ward and S.A. Yost, Mod. Phys. Lett. A 19(2004) 2113; B.F.L. Ward, C. Glosser, S. Jadach and S.A. Yost, in Proc. DPF 2004, Int. J. Mod. Phys. A 20 (2005) 3735; in Proc. ICHEP04, vol. 1, eds. H. Chen et al.,(World. Sci. Publ. Co., Singapore, 2005) p. 588; B.F.L. Ward and S. Yost, preprint BU-HEPP-05-05, in Proc. HERA-LHC Workshop, CERN-2005-014; in Moscow 2006, ICHEP, vol. 1, p. 505; Acta Phys. Polon. B 38 (2007) 2395; arXiv:0802.0724,

PoS(RADCOR2007)(2007) 038; B.F.L. Ward et al., arXiv:0810.0723, in Proc. ICHEP08; arXiv:0808.3133, in Proc. 2008 HERA-LHC Workshop,DESY-PROC-2009-02, eds. H. Jung and A. De Roeck, (DESY, Hamburg, 2009)pp. 180-186, and references therein.

[2] See for example S. Jadach et al., in Physics at LEP2, vol. 2, (CERN, Geneva, 1995) pp. 229-298.

[3] B.F.L. Ward, S.K. Majhi and S.A. Yost, PoS(RADCOR 2011) (2012) 022.

[4] G. Altarelli and G. Parisi, Nucl. Phys. B126 (1977) 298; Yu. L. Dokshitzer, Sov. Phys. JETP 46 (1977) 641; L. N. Lipatov, Yad. Fiz. 20 (1974) 181; V. Gribov and L. Lipatov, Sov. J. Nucl. Phys. 15 (1972) 675, 938; see also J.C. Collins and J. Qiu, Phys. Rev. D39 (1989) 1398.

[5] C.G. Callan, Jr., Phys. Rev. D2 (1970) 1541; K. Symanzik, Commun. Math. Phys. 18 (1970) 227, and in Springer Tracts in Modern Physics, 57, ed. G. Hoehler (Springer, Berlin, 1971) p. 222; see also S. Weinberg, Phys. Rev. D8 (1973) 3497.

[6] D. R. Yennie, S. C. Frautschi, and H. Suura, Ann. Phys. 13 (1961) 379; see also K. T. Mahanthappa, Phys. Rev. 126 (1962) 329, for a related analysis.

[7] S. Jadach and B.F.L. Ward, Phys. Rev. D38 (1988) 2897;ibid. D39 (1989) 1471; ibid. D40 (1989) 3582; S.Jadach, B.F.L. Ward and Z. Was, Comput. Phys. Commun. 66 (1991) 276; S.Jadach and B.F.L. Ward, Phys. Lett. B274 (1992) 470; S. Jadach et al., Comput. Phys. Commun. 70 (1992) 305; S.Jadach, B.F.L. Ward and Z. Was, Comput. Phys. Commun. 79 (1994) 503; S. Jadach et al., Phys. Lett. B353 (1995) 362; ibid. B384 (1996) 488; Comput. Phys. Commun. 102 (1997) 229; S.Jadach, W. Placzek and B.F.L. Ward, Phys. Lett. B390 (1997) 298; Phys. Rev. D54 (1996) 5434; Phys.Rev. D56 (1997) 6939; S.Jadach, M. Skrzypek and B.F.L. Ward, Phys. Rev. D55 (1997) 1206; See, for example, S. Jadach et al., Phys. Lett. B417 (1998) 326; Comput. Phys. Commun. 119 (1999) 272; Phys. Rev. D61 (2000) 113010; Phys. Rev. D65 (2002) 093010; Comput. Phys. Commun. 140 (2001) 432, 475; S.Jadach, B.F.L. Ward and Z. Was, Comput. Phys. Commun. 124 (2000) 233; and references therein.

[8] B.F.L. Ward, Adv. High Energy Phys. 2008 (2008) 682312.

[9] B.F.L. Ward, Ann. Phys. 323 (2008) 2147. 
[10] B.F.L. Ward, Mod. Phys. Lett. A17 (2002) 2371; Open Nucl. Part. Phys. J 2 (2009) 1; J. Cos. Astropart. Phys.0402 (2004) 011; Mod. Phys. Lett. A23 (2008) 3299.

[11] S. Joseph et al., Phys. Lett. B685 (2010) 283; Phys. Rev. D81 (2010) 076008; S. Majhi et al., Phys. Lett. B719 (2013) 367; arXiv:1305.0023.

[12] G. Sterman,Nucl. Phys. B281, 310 (1987); S. Catani and L. Trentadue, Nucl. Phys. B327, 323 (1989); ibid. B353, 183 (1991).

[13] See for example C. W. Bauer, A.V. Manohar and M.B. Wise, Phys. Rev. Lett. 91 (2003) 122001; Phys. Rev. D70 (2004) 034014; C. Lee and G. Sterman, Phys. Rev. D 75 (2007) 014022.

[14] S. Majhi et al., to appear.

[15] J.C. Collins and D.E. Soper, Nucl. Phys. B193 (1981) 381; ibid.213(1983) 545; ibid. 197(1982) 446; J.C. Collins, D.E. Soper and G. Sterman, Nucl. Phys. B250(1985) 199; in Les Arcs 1985, Proceedings, QCD and Beyond, pp. 133-136.

[16] C. Balazs and C.P. Yuan, Phys. Rev. D56 (1997) 5558; G.A. Ladinsky and C.P. Yuan, Phys. Rev. D50 (1994) 4239; F. Landry et al., Phys. Rev. D67 (2003) 073016.

[17] F. Bloch and A. Nordsieck, Phys. Rev. 52 (1937) 54.

[18] G. Corcella et al., hep-ph/0210213; J. High Energy Phys. 0101 (2001) 010; G. Marchesini et al., Comput. Phys. Commun.67 (1992) 465.

[19] M. Bahr et al., arXiv:0812.0529 and references therein.

[20] T. Sjostrand, S. Mrenna and P. Z. Skands, Comput. Phys. Commun. 178 (2008) 852-867.

[21] T. Gleisberg et al., J.High Energy Phys. 0902 (2009) 007.

[22] P. Nason, J. High Energy Phys. 0411 (2004) 040.

[23] S. Frixione and B.Webber, J. High Energy Phys. 0206 (2002) 029; S. Frixione et al., arXiv:1010.0568.

[24] S. Chatrchyan et al., arXiv:1110.4973; Phys. Rev. D85 (2012) 032002.

[25] G. Aad et al., arXiv:1107.2381; Phys. Lett. B705 (2011) 415.

[26] V.M. Abasov et al., Phys. Rev. Lett. 100 (2008) 102002.

[27] C. Galea, in Proc. DIS 2008, London, 2008, http://dx.doi.org/10.3360/dis.2008.55.

[28] R.P. Feynman, M. Kislinger and F. Ravndal, Phys. Rev. D3 (1971) 2706; R. Lipes, ibid.5 (1972) 2849; F.K. Diakonos, N.K. Kaplis and X.N. Maintas, ibid. 78 (2008) 054023; K. Johnson, Proc. Scottish Summer School Phys. 17 (1976) p. 245; A. Chodos et al., Phys. Rev. D9 (1974) 3471; ibid. 10 (1974) 2599; T. DeGrand et al., ibid. 12 (1975) 2060.

[29] J. Bjorken, in Proc. 3rd International Symposium on the History of Particle Physics: The Rise of the Standard Model, Stanford, CA, 1992, eds. L. Hoddeson et al. (Cambridge Univ. Press, Cambridge, 1997) p. 589, and references therein.

[30] S. Jadach, B.F.L. Ward and Z. Was, arXiv:1307.4037; Phys. Rev. D, 2013, in press.

[31] D. Bardin et al., JETP Lett. 96 (2012) 285; arXiv:1207.4400; S.G. Bondarenko and A.A. Sapronov, arXiv:1301.3687; L. Barze et al., arXiv:1302.4606; C.M. Carloni-Calame et al., J. High Energy Phys. 05 (2005) 019; G. Balossini et al., J. Phys. Conf. Ser. 110 (2008) 042002; Y. Li and F. Petriello, Phys. Rev. D86(2012) 094034; V. A. Zykunov, Eur. Phys. J. C3 (2001) 9; S. Dittmaier and M. Kramer, Phys. Rev. D65 (2002) 073007; S. Dittmaier and M. Huber, J. High Energy Phys.1001 (2010) 060; A. Denner et al., J. High Energy Phys. 1106 (2011) 069; C. Bernaciak and D. Wackeroth, Phys. Rev. D85 (2012) 093003; and references therein. 
[32] S. Hassani, in Proc. Recntres de Moriond EW, 2013, in press; H. Yin, ibid., 2013, in press; G. Aad et $a l .$, arXiv:1211.6899, and references therein.

[33] B.F.L. Ward, arXiv:1008.1046; PoS(ICHEP10)(2010) 477; Phys. Dark Univ.2(2013) 97.

[34] A.G. Riess et al., Astron. Jour. 116 (1998) 1009; S. Perlmutter et al., Astrophys. J. 517 (1999) 565.

[35] C. Amsler et al., Phys. Lett. B667 (2008) 1.

[36] M. Reuter, Phys. Rev. D57 (1998) 971; O. Lauscher and M. Reuter, ibid. 66 (2002) 025026; A. Bonanno and M. Reuter, ibid. 62 (2000) 043008, and references therein.

[37] A. Bonanno and M. Reuter, Phys. Rev. D65 (2002) 043508; Jour. Phys. Conf. Ser. 140 (2008) 012008, and references therein.

[38] V. Branchina and D. Zappala, G. R. Gravit. 42 (2010) 141; arXiv:1005.3657, and references therein.

[39] J. Sola, J. Phys. A41 (2008) 164066.

[40] See for example G. Stiegman, Ann. Rev. Nucl. Part. Sci.57 (2007) 463, and references therein.

[41] See P.S. Bhupal Dev and R.N. Mohapatra, Phys. Rev. D82 (2010) 035014, and references therein.

[42] S. Basilakos, M. Plionis and J. Sola, arXiv:0907.4555; J. Grande et al., J. Cos. Astropart. Phys. 1108 (2011) 007, arXiv:1103.4632; H. Fritzsch and J. Sola, arXiv:1202.5097; and references therein. 